\title{
Publicidad alimentaria en horario infantil: análisis de los anuncios emitidos en tres canales televisivos
}

Cristina Gil González - Universidad de Zaragoza

Ángel Luis Cortés Gracia - Universidad de Zaragoza

Recepción: 17.12.2019 | Aceptado: 10.01.2020

Correspondencia a través de ORCID: Cristina Gil González

Citar: Gil González, C y Cortés Gracia, AL (2020). Publicidad alimentaria en horario infantil: análisis de los anuncios emitidos en tres canales televisivos. REIDOCREA, 9, 01-10.
0000-0002-5618-3390

0000-0002-7075-9683

Financiación y agradecimientos: Grupo de referencia BEAGLE Investigación en Didáctica de las Ciencias Naturales (Gobierno de Aragón y Fondo Social Europeo) Instituto de Investigación en Ciencias Ambientales de Aragón (IUCA/UNIZAR). Proyecto EDU2016-76743-P (MINECO).

\begin{abstract}
Resumen: Los medios de comunicación, y especialmente la televisión, ejercen una gran presión para que se consuman cada vez más alimentos industriales, lo que está contribuyendo a agravar la epidemia de obesidad infantil existente hoy en día en nuestra sociedad. El objetivo principal de este estudio es determinar qué anuncios con publicidad alimentaria aparecen en tres canales de televisión dirigidos específicamente al público infantil y, posteriormente, analizar su contenido. El estudio se centra en tres franjas horarias durante tres periodos distintos de quince días consecutivos. El análisis de contenido aborda cuatro aspectos relevantes desde una perspectiva educativa: 1) tipo de alimento, 2) mensaje general del anuncio, 3) contenido científico asociado y 4) contexto o contenido educativo vinculado. Los resultados muestran hasta 20 marcas de productos alimentarios manifestadas en un total de 36 anuncios distintos, emitidos principalmente en horario de 17 a $19 \mathrm{~h}$ y de 20:30 a 22h. Destaca el uso de personajes famosos en la tercera parte de los anuncios (12 de 36), mensajes con contenido científico en 19 de ellos y referencias explícitas al contexto escolar en tan solo 3 anuncios. Muchos de estos contenidos no cumplen con el Código PAOS y podrían condicionar una correcta educación alimentaria.
\end{abstract}

Palabras clave: Publicidad televisada

Food advertising in child schedule: analysis of the advertisements broadcast on three television channels

\begin{abstract}
The media, and especially television, perform a great pressure to consume more and more industrial foods, which is contributing to aggravate the epidemic of childhood obesity that exists today in our society. The main objective of this study is determinate what ads with food advertising appear in three channels of TV aimed at children public and, later, analyze their content. The study is focused on three time slots for three different periods of fifteen consecutive days. The content analysis involves four relevant aspects from an educational perspective: 1) type of food, 2) general message of advertisement, 3) scientific content associated and 4) context or educational content linked. The results show 20 brands of food products stated in a total of 36 different advertisements, mainly issued from 17-19h and from 20:30-22h. The use of famous characters stands out in the third part of ads (12 of 36), message with scientific content in 19 of them and explicit references to escolar context in only 3 ads. A lot of these contents don't comply with PAOS Code and they could affect a correct food education.
\end{abstract}

Keywords: Television advertising

\section{Introducción}

La obesidad infantil representa en la actualidad uno de los principales problemas de salud pública. Aunque Estados Unidos es el país del mundo más afectado por lo que ya se considera una epidemia, España es uno de los países europeos con mayor prevalencia del sobrepeso y la obesidad infantil (Fernández-Gómez y Díaz-Campo, 2014; Casas Esteve y Gómez Santos, 2016). Las causas de este incremento son múltiples, aunque algunas investigaciones destacan la enorme presión que los medios de comunicación, y en especial la televisión, ejercen para que se consuman cada vez más alimentos industriales (León-Flández, 2017). A pesar de que la OMS recomienda 
reducir el impacto negativo que está produciendo en nuestra sociedad la publicidad de alimentos ricos en grasas, azúcares y sales (Bosqued et al., 2016), buena parte de los anuncios que se emiten en horario infantil siguen promocionando alimentos y bebidas altos en grasas saturadas, ácidos grasos trans, azúcares o sal (también conocidos como AGATAS). Autores como Tirado et al. (2004) añaden que esos anuncios televisivos favorecen la inclusión en la dieta de ese tipo de alimentos utilizando estrategias de marketing que usan mensajes del tipo "sin colesterol", "sin azúcar" o "sin grasas vegetales" para ocultar su verdadero contenido nutricional.

La infancia es una etapa clave en relación con la publicidad alimentaria. Los niños, especialmente los más pequeños, representan un grupo de edad acrítico y fácil de manipular, por lo que sus preferencias, peticiones de compra y hábitos alimentarios están siendo modificados al ser incapaces de entender la intención de la publicidad y aceptando las afirmaciones publicitarias como hechos (Bosqued et al., 2016; Cabello et al., 2016). El interés de las industrias alimentarias por los niños radica en el elevado número de horas que pasan frente al televisor, su influencia en las compras familiares y su papel como futuros consumidores. El efecto de los anuncios de alimentos en televisión afecta a niños y adultos, ya que una gran proporción de los mismos están dirigidos a las familias. Sin embargo, en muchas ocasiones, las técnicas de marketing que enmascaran productos no saludables no son percibidas por los espectadores (Díaz Ramírez et al., 2011; Bacardí Gascón y Jiménez Cruz, 2015). Herrero Aguado (2008) destaca algunos elementos de seducción utilizados en este tipo de publicidad, como un timbre de voz adecuado, la presencia de otros niños en el anuncio o incluso la presencia de animales. De esta manera los niños memorizan los eslóganes de las marcas, que les permiten disfrutar con la palabra y el ritmo. En 2006, el Instituto de Medicina de Estados Unidos informó de que existían pruebas suficientes para establecer una relación causal entre publicidad televisiva y obesidad (Gootman, McGinnis y Kraak, 2006). Un estudio reciente muestra resultados similares en el caso de la población infantil española (Bawake et al., 2019). Esto afecta especialmente a las preferencias de alimentos y bebidas, solicitudes de compra y consumo a corto plazo entre los niños de 2 a 11 años (Cabello et al., 2016). De hecho, González y López (2012) y Santos (2015) indican que muchos padres y madres reconocen que sus hijos e hijas piden determinados alimentos directamente influenciados por los anuncios de televisión.

A todo lo anterior debemos añadir la presión publicitaria existente en el tiempo de protección reforzada para la infancia, así como durante la propia programación infantil (Bosqued et al., 2016). Herrero Aguado (2008) ya señalaba que, en determinados momentos del año, la publicidad de alimentos representaba la mitad de los anuncios emitidos en horario infantil. En ese sentido hay que añadir que algunas investigaciones muestran como las marcas más anunciadas en estos periodos corresponden sobre todo a productos considerados como no saludables (Romero-Fernández, Royo-Bordonada y Rodríguez-Artalejo, 2013; Bosqued et al., 2016). Esta realidad es común a muchos otros países, como demuestran los resultados de Vik et al. (2013), que indican además que las probabilidades de sobrepeso son menores en niños que no ven nunca la televisión durante el almuerzo y la cena. De esta forma, algunos estudios muestran como un alto porcentaje de los niños desearían desayunar y merendar de forma no saludable (más del $80 \%$, según Teruel Benítez, 2014) a pesar de que en su vida cotidiana seguían hábitos saludables en estas comidas.

A partir de las recomendaciones de la Organización Mundial de la Salud (OMS) sobre la promoción de alimentos y bebidas no alcohólicas dirigida a niños, en España se optó por la autorregulación publicitaria (Martín, Fernández y Ortiz, 2011). Así se desarrollaron varios códigos sobre contenidos televisivos derivados de la Estrategia NAOS (AESAN, 2005), destacando el Código PAOS (AECOSAN, 2012). En este código se señala, por ejemplo, que "la publicidad de alimentos o bebidas dirigida a menores de hasta 12 años 
en ningún caso explotará la especial confianza de estos niños, en sus padres, en profesores, o en otras personas, tales como profesionales de programas infantiles, 0 personajes (reales o ficticios) de películas o series de ficción" (norma 14); "no se llevarán a cabo telepromociones de productos alimenticios o bebidas con ocasión de programas dirigidos a menores de hasta 12 años" (norma 15). En estos momentos, los anuncios del sector de alimentos y bebidas suponen un $20 \%$ del total de sectores publicitarios y, dentro de ese sector concreto, la publicidad dirigida a niños de 4-12 años representa en torno a un 13\% (AECOSAN, 2019). Buena parte de la publicidad dirigida a ese grupo de edad se emite (y visualiza) en horario de protección reforzada para la infancia $(27,5 \%$ del total), por lo que se supone que ese tipo de publicidad va a tener un importante impacto en el público al que va dirigida. Recientes estudios (Royo-Bordonada et al., 2016; León-Flández et al., 2017) muestran los desajustes existentes entre el Código PAOS y los contenidos de muchos anuncios de años anteriores, destacando los incumplimientos normativos y alegaciones sobre nutrición y salud que implicaban a productos considerados como poco saludables.

\section{Objetivos}

Ante la situación descrita, y como paso previo al diseño de propuestas educativas concretas que ayuden de frenar los problemas que la obesidad infantil puede generar en nuestra sociedad, parece oportuno determinar qué tipo de publicidad sobre alimentos y bebidas ofrecen los operadores televisivos que programan contenidos destinados directamente al público infantil (4-12 años). Dado que en estos momentos existen diversas plataformas televisivas digitales con canales dirigidos específicamente al grupo de edad señalado, se prestará especial atención a los canales correspondientes a operadores no adheridos al convenio del Código PAOS.

Así, el objetivo principal de este trabajo consistirá en determinar qué anuncios con publicidad sobre alimentos y bebidas aparecen en las principales franjas horarias de tres canales dirigidos específicamente al público infantil. Una vez determinados los mismos, se procederá al análisis de su contenido centrado en cuatro aspectos relevantes desde el punto de vista educativo: 1) tipo de alimento, 2) mensaje general del anuncio, 3) contenido científico asociado al producto o marca y 4) contexto o contenido educativo vinculado.

\section{Métodos}

Para identificar los anuncios y las marcas de los productos alimentarios que se emiten en la televisión se ha procedido a realizar una visualización de varios canales con programación infantil en tres franjas horarias distintas. Según estudios previos, estos canales y horarios son los que presentan más publicidad alimentaria, influyen en los hábitos y decisiones de compra de los niños, al estar especialmente dirigidos a este grupo de población, y en esa franja alcanzan su mayor audiencia (Busquet y Reinares 2009; Caroli et al., 2004; Jiménez, 2006; Kelly et al., 2010; OCU, 2010; RomeroFernández et al., 2013).

Los canales de televisión dirigidos hacia el público infantil para los que se ha realizado una visualización previa han sido Neox, Clan TV, Boing, Disney Channel (HD), Disney XD, Disney Junior, Nickelodeon, Canal Panda, Nick JR y Baby TV. De todos ellos, Neox, Clan TV, Boing y Disney Channel son los que más audiencia consiguen en los últimos estudios, según los análisis mensuales de comportamiento de la audiencia de televisión y los resultados del Estudio General de Medios 2019 (https://www.barloventocomunicacion.es; https://www.aimc.es). No obstante, Neox se ha descartado para el análisis, ya que no es un canal representativo del público comprendido entre 4 y 12 años (target 4-12) y tan sólo muestra contenido específico 
para el público infantil en su espacio Neox Kids, en el que agrupa series como "Shin Chan", "Los Padrinos Mágicos" o "Angry Birds". Por su parte, Clan TV pertenece a la Corporación RTVE y, como cadena de carácter público, no puede emitir publicidad comercial. Esta ausencia de publicidad también ocurre en Canal Panda y Disney XD, por lo que igualmente se han descartado para su análisis. Finalmente, los canales Baby TV, Disney Junior y Nick JR se han descartado por mostrar un contenido dirigido hacia un público infantil de edades muy tempranas (menores de 4 años).

De esta manera, los canales comerciales que se han seleccionado por presentar contenidos específicos para un público infantil de edades comprendidas entre los 4 y 12 años han sido Boing (10,9-11,3\% de audiencia en el grupo 4-12 en 2018-2019), Disney Channel (10,3-11,3\%) y Nickelodeon (primera cadena del grupo 4-12 entre las temáticas de pago). Su visualización se ha realizado en distintas franjas horarias, siendo éstas 13$15 \mathrm{~h}, 17-19 \mathrm{~h}$ y $20: 30-22 \mathrm{~h}$, a lo largo de tres periodos de quince días consecutivos durante los meses de noviembre de 2018 y marzo y julio de 2019 (unas 750 horas de contenidos televisivos). La selección de estos meses se debe a que recogen dos periodos durante el curso escolar y un periodo durante el tiempo vacacional. En la selección de las franjas horarias a analizar no se ha considerado el horario del desayuno puesto que no sigue un criterio uniforme en perspectivas de tarifas comerciales y audiencias (Estudio General de Medios 2019, en https://www.aimc.es). El registro de toda la información se ha llevado a cabo mediante la grabación completa de vídeo de las franjas horarias señaladas en cada canal (plataforma digital) y la extracción de los anuncios con publicidad alimentaria para su posterior análisis.

El análisis de contenido (Bardin, 1986), independientemente del tipo de documento analizado, "sólo puede entenderse de una forma completa si lo situamos en el contexto de un diseño de investigación" (López Noguero, 2002, p. 173). De esta forma, se deben diseñar plantillas ad hoc y definir unidades de análisis y categorías que dependerán tanto del formato documental como de los objetivos de la investigación (Boronat Mundina, 2005; Moreno Castro, 2006; Torres García et al., 2017). Para analizar toda la información recogida se han diseñado unas plantillas de registro de elaboración propia donde se han anotado diariamente, por meses y en las distintas franjas horarias, las marcas de los productos alimentarios que aparecen en los anuncios emitidos en cada canal de televisión (tabla 1). Además, se ha detallado el contenido que presentan los mismos referentes a la presencia de elementos persuasivos (sonidos, personajes famosos y premios), mensaje general transmitido, imágenes y colores, mensajes con contenido científico y posible relación con el contexto escolar (tabla 2).

Tabla 1. Plantilla de análisis usada para registrar las marcas alimentarias. En este ejemplo se muestran las marcas que han aparecido en el canal de televisión infantil Boing durante algunos días del mes de marzo de 2019.

\begin{tabular}{|c|c|c|}
\hline \multicolumn{3}{|c|}{ BOING } \\
\hline & MARZO & \\
\hline DÍA & \multicolumn{2}{|c|}{$16 / 03 / 2019$} \\
\hline 13-15H & 17-19H & $20: 30-22 \mathrm{H}$ \\
\hline COLACAO & $\begin{array}{c}\text { LA PIARA } \\
\text { BABYBEL } \\
\text { TOSTARICA } \\
\text { MCDONALD'S } \\
\text { BAZOOKA } \\
\text { DON SIMÓN }\end{array}$ & $\begin{array}{c}\text { BAZOOKA } \\
\text { DON SIMÓN } \\
\text { TOSTARICA } \\
\text { BABYBEL } \\
\text { LA PIARA }\end{array}$ \\
\hline DÍA & \multicolumn{2}{|c|}{$17 / 03 / 2019$} \\
\hline $13-15 \mathrm{H}$ & $17-19 \mathrm{H}$ & $20: 30-22 \mathrm{H}$ \\
\hline COLACAO & $\begin{array}{l}\text { LA PIARA } \\
\text { TOSTARICA } \\
\text { COLACAO } \\
\text { BABYBEL } \\
\text { GUSANITOS }\end{array}$ & $\begin{array}{l}\text { DON SIMÓN } \\
\text { LA PIARA } \\
\text { TOSTARICA } \\
\text { COLACAO } \\
\text { BAYBEL }\end{array}$ \\
\hline DIA & \multicolumn{2}{|c|}{$18 / 03 / 2019$} \\
\hline $13-15 \mathrm{H}$ & $17-19 \mathrm{H}$ & $20: 30-22 \mathrm{H}$ \\
\hline
\end{tabular}




\begin{tabular}{c|c|c}
\hline ALDI S.A & DON SIMÓN & COLACAO \\
\hline BABYBEL & TOSTARICA & TOSTARICA \\
COLACAO & ALDI S.A & ALDI S.A \\
& DABYBEL SIMÓN & BABYBEL \\
& MCDONALD'S & BAZOOKA \\
\hline
\end{tabular}

Tabla 2. Plantilla de análisis utilizada para analizar el contenido del anuncio. Ejemplo: Helado "LlaoLlao".

ANUNCIO
SONIDO
PERSONAJES FAMOSOS
GANAS UN REGALO

\section{Resultados}

Tras la visualización de todos los contenidos, se han identificado hasta 20 marcas diferentes de productos alimentarios que han aparecido en alguna de las tres cadenas analizadas (tabla 3). Los resultados por marcas se han organizado por meses y canales de televisión, así como por franjas horarias de emisión (tabla 4).

Del listado de marcas alimentarias que han aparecido en cada mes en cada uno de los canales infantiles estudiados, destaca el acusado aumento que se produce en el número de marcas alimentarias entre noviembre y julio, siendo tres en noviembre de 2018, 11 en marzo de 2019 y 14 en julio de 2019 (tabla 4). Así mismo, hay que señalar que McDonald's es la única marca que se emite en los tres periodos estudiados, mientras la publicidad de las otras dos marcas emitidas en noviembre (Choco Krispies y Lanjarón) desaparece en los meses de marzo y julio de 2019. Algunas marcas como Gallina Blanca, Gusanitos, La Piara y Strings and Things aparecen exclusivamente en el mes de marzo, mientras las marcas Frigo, Hero Baby, Lidl, LlaoLlao, Phoskitos, Puleva y Vichy Catalán aparecen solo en julio. Los resultados muestran también que hay seis marcas que se anuncian tanto en el mes de marzo como en julio, como es el caso de Aldi S.A, Babybel, Bazooka, Colacao, Don Simón y TostaRica, aunque no en todas las cadenas analizadas.

Destaca el hecho de que Colacao y TostaRica son las dos únicas marcas presentes en todos los canales y franjas del mes de marzo, mientras que Colacao, Frigo, McDonald's y Phoskitos son las marcas que se emiten en todos los canales y franjas horarias analizadas en julio. Además, hay que señalar que los canales Boing y Disney contienen publicidad de hasta 14 marcas alimentarias distintas, alcanzando su máximo en la franja horaria de 17-19h del mes de julio. El canal Nickelodeon es el que menos marcas alimentarias publicita de los tres analizados, mostrando un máximo de cinco marcas distintas en los tres tramos horarios del mes de julio. 
Tabla 3. Marcas alimentarias que han aparecido en los tres canales de televisión infantiles analizados.

\begin{tabular}{c} 
MARCAS ALIMENTARIAS \\
ALDI S.A. \\
BABYBEL (GRUPO BEL FOODSERVICE) \\
BAZOOKA (GRUPO BAZOOKA CANDY BRANDS) \\
CHOCO KRISPIES (GRUPO KELLOGG'S) \\
COLACAO (GRUPO IDILIA FOODS) \\
DON SIMÓN (GRUPO GARCIA CARRIÓN) \\
FRIGO (GRUPO FRIGO) \\
\hline GALLINA BLANCA (GRUPO AGROLIMEN) \\
GUSANITOS (GRUPO RISI) \\
HERO BABY (GRUPO HERO) \\
LANJARÓN (GRUPO DANONE) \\
LA PIARA (GRUPO ADAM FOODS) \\
\hline LIDL STIFTUNG \& CO. KG (GRUPO SCHWARZ) \\
LLAOLLAO S.L. \\
MCDONALD'S \\
\hline PHOSKITOS (GRUPO ADAM FOODS) \\
PULEVA (GRUPO LACTALIS) \\
STRINGS AND THINGS (GRUPO KERRY) \\
TOSTARICA (CUÉTARA, GRUPO ADAM FOODS) \\
VICHY CATALÁN (GRUPO VICHY CATALÁN)
\end{tabular}

Tabla 4. Marcas alimentarias anunciadas por meses, franjas horarias y canales de TV.

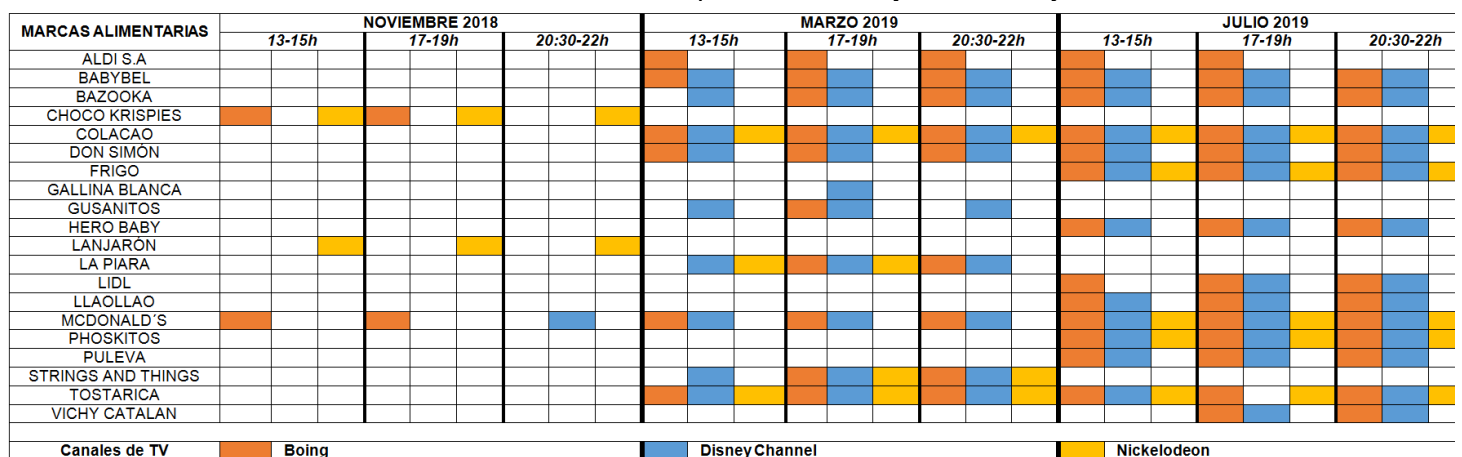

Por otro lado, se han analizado en detalle todos los anuncios con publicidad alimentaria emitidos por las distintas marcas en esos periodos (un total de 36 anuncios, tabla 5). En primer lugar, se han identificado los productos concretos anunciados en cada uno de ellos junto con la marca alimentaria a la que pertenecen $y$, posteriormente, se ha realizado un análisis de contenido de estos a partir de la información recogida en las plantillas de registro elaboradas a tal efecto.

Tabla 5. Resultados del análisis de contenido de los anuncios publicitados en los tres canales de televisión infantiles estudiados. Se señala en color crema la presencia de los distintos tipos de contenido en cada anuncio.

\begin{tabular}{|c|c|c|c|c|c|c|c|c|c|c|c|}
\hline \multirow{2}{*}{$\begin{array}{l}\text { PRODUCTOS } \\
\text { ALLMENTARIOS }\end{array}$} & \multirow[b]{2}{*}{ Anuncios } & \multirow[b]{2}{*}{$\begin{array}{l}\text { MARCA } \\
\text { ALIMENTARIA }\end{array}$} & \multicolumn{7}{|c|}{$\begin{array}{l}\text { ELEMENTOS PERSUASIVOS } \\
\text { Sonido }\end{array}$} & \multirow{3}{*}{$\begin{array}{l}\text { MENSAJE CON } \\
\text { CONTENIDO } \\
\text { CIENTIFICO }\end{array}$} & \multirow{2}{*}{$\begin{array}{c}\text { RELACION CON EL } \\
\text { CONEXTO } \\
\text { ESCOLAR }\end{array}$} \\
\hline & & & $\begin{array}{l}\text { Personales } \\
\text { tamosos }\end{array}$ & $\begin{array}{l}\text { Voz adura } \\
\text { mascrumna }\end{array}$ & $\begin{array}{l}\text { Voz adulta } \\
\text { trmenana }\end{array}$ & 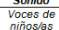 & 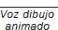 & Müsclca & $\begin{array}{l}\text { Ganas in } \\
\text { ragalo }\end{array}$ & & \\
\hline AGUA & AGUA LANJARÓN & LANIARON & & & & & & & & & \\
\hline BATDOS & BATIDOS PULEVA & PULEVA & & & & & & & & & \\
\hline BEBIDAS SIN GAS & BEBIDA VICHY CATALAN & VICHY CATALAN & & & & & & & & & \\
\hline \multirow[t]{2}{*}{ BoLlos } & $\begin{array}{l}\text { PHOSKITOS } 1 \\
\text { PSOTOS }\end{array}$ & PHOSKITOS & & & & & & & & & \\
\hline & $\frac{P O C S R T O S 2}{\text { COLACAO } 1}$ & 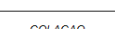 & & & & & & & & & \\
\hline CACAO & COLACAO 2 & COLACAO & & & & & & & & & \\
\hline caramelos & 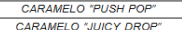 & ВАZООКА & & & & & & & & & \\
\hline CEREALES & $\begin{array}{l}\text { CAAREALELS CHOCO KRISPIIES } \\
\text { CERES }\end{array}$ & CHOCO KRISPIES & & & & & & & & & \\
\hline \multirow{2}{*}{ galletas } & $\begin{array}{l}\text { GALLETAS TISTAAICA } \\
\text { CLISICAS }\end{array}$ & & & & & & & & & & \\
\hline & $\begin{array}{l}\text { GALLETAS IOSTARICA } \\
\text { OOEANX }\end{array}$ & TOSTARICA & & & & & & & & & \\
\hline \multirow{3}{*}{ HAMBURGUESAS } & $\begin{array}{l}\text { MC DONALD'S1 } \\
\text { MC DONALD'S 2 }\end{array}$ & & & & & & & & & & \\
\hline & $\begin{array}{l}\text { MC DONALD'S } 3 \\
\text { MC DONALD'S } 4\end{array}$ & MC DONALO'S & & & & & & & & & \\
\hline & MC DONALD'S 5 & & & & & & & & & & \\
\hline \multirow[b]{2}{*}{ HELADOS } & $\begin{array}{c}\text { MC DONALL'S } 6 \\
\text { HELADO "PRIILO CHUCHES" }\end{array}$ & & & & & & & & & & \\
\hline & HELADO "LLAOLLAO" & LLAOLLAO & & & & & & & & & \\
\hline \multirow{2}{*}{$\begin{array}{l}\frac{P A S T A}{\text { PATA }} \\
\text { PATE }\end{array}$} & YATEKOMO & GALLINA BLANCA & & & & & & & & & \\
\hline & PATE LA PIARA & LAPIARA & & & & & & & & & \\
\hline \multirow{2}{*}{ QUESOS } & 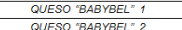 & BABYBEL & & & & & & & & & \\
\hline & $\begin{array}{l}\text { QUESO "BABYEEL" } 2 \\
\text { QUESO "CHEESTRINGS" }\end{array}$ & $\begin{array}{l}\text { STRINGS AND } \\
\text { STHNOSD }\end{array}$ & & & & & & & & & \\
\hline \multirow{2}{*}{ SNACKS } & HERO GUISANTES & HERO BABY & & & & & & & & & \\
\hline & $\begin{array}{l}\text { HERO LENTEAS } \\
\text { GUSANTIOS }\end{array}$ & GUSANITOS & & & & & & & & & \\
\hline \multirow{4}{*}{ VARIOS } & SUPERMERCADO ALDI 1 & & & & & & & & & & \\
\hline & 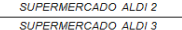 & ALDIS.A & & & & & & & & & \\
\hline & 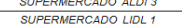 & & & & & & & & & & \\
\hline & SUPERMERCADO LIDL 2 & CO. KG & & & & & & & & & \\
\hline zumos & $\begin{array}{l}\text { DON SMIMON } \\
\text { SINOOIIEE }\end{array}$ & DON SMON & & & & & & & & & \\
\hline
\end{tabular}


Del análisis de contenido de los anuncios, destaca en primer lugar el uso de personajes famosos en un tercio de estos (12 de 36), a pesar de que el Código PAOS señala explícitamente que no se explotará la especial confianza de los niños y niñas de esas edades en esos personajes (reales o de ficción). Resulta llamativa la aparición de los mismos personajes de ficción en anuncios de distintas marcas alimentarias, como los personajes de "La Patrulla Canina" en los anuncios de agua Lanjarón y helado LlaoLlao y los personajes de "Pokémon" en los anuncios de McDonald's y Phoskitos. Además, la obtención de un regalo con la compra del producto se ofrece en 15 de los anuncios analizados (10 de ellos también usaban personajes famosos como reclamo). En cuanto al resto de los elementos persuasivos, en 27 de los anuncios aparece una voz adulta masculina, frente a 14 anuncios con voces de dibujos animados, 10 con voces de niños/niñas y 8 con una voz femenina. Tan solo los anuncios Hero Guisantes y Hero Lentejas no incluyen la música como elemento persuasivo.

Los mensajes con contenido científico (19 anuncios) son similares entre anuncios de productos de distintas marcas alimentarias y prácticamente iguales en los anuncios de productos que pertenecen a una misma marca alimentaria (tabla 6). Por ejemplo, en los anuncios de las marcas alimentarias Puleva, Choco Krispies y Vichy Catalán y algunos de McDonald's se hace referencia a la ausencia de azúcares añadidos o al reducido contenido de azúcar añadido que contienen, mientras que en varios de los anuncios de la marca McDonald's se repite el mensaje "toma frutas y verduras cada día". Varias marcas recurren a la introducción de porcentajes de algún tipo (30\% menos de azúcar, $90 \%$ de leche, $100 \%$ ecológicos, $100 \%$ exprimido), usan términos relacionados con los componentes que tienen (calcio, vitaminas, hierro, proteínas), resaltan los que no tienen (sin sal, sin gas, sin azúcar, sin conservantes, sin colorantes, no fritos) o incluyen las etiquetas BIO y ECO (Hero y Aldi S.A). Por otro lado, varios anuncios recurren a mensajes que aluden directamente a cuestiones fisiológicas, como Puleva (necesarios para el crecimiento y el desarrollo normal de los huesos; contribuyen a conservar la masa muscular) o Simon Life (contribuyen a la protección de las células frente al daño oxidativo). Finalmente, estas últimas marcas también introducen afirmaciones categóricas relacionadas con la salud en su publicidad, como Puleva (ningún batido es más saludable) y Simon Life (es sano y natural).

Tabla 6. Mensajes con contenido científico que aparecen en los anuncios de productos alimentarios emitidos en los tres canales de televisión infantiles analizados.

\begin{tabular}{|c|c|c|}
\hline MARCA ALIMENTARIA & ANUNCIOS & MENSAJE CON CONTENIDO CIENTÍFICO \\
\hline PULEVA & BATIDOS PULEVA & $\begin{array}{l}\text { - } \quad 30 \% \text { menos de azúcar añadido } \\
\text { - } \quad 90 \% \text { leche } \\
\text { - } \quad \text { Ningún batido es más saludable y la vitamina } D \text { son necesarios para el } \\
\text { - } \quad \text { crecimiento y el desarrollo normal de los huesos } \\
\text { - } \quad \text { Las proteínas contribuyen a conservar la masa muscular }\end{array}$ \\
\hline CHOCO KRISPIES & CEREALES CHOCO KRISPIES & - $\quad 30 \%$ menos de azúcares \\
\hline TOSTARICA & $\begin{array}{l}\text { GALLETAS TOSTARICA } \\
\text { OCEANIX }\end{array}$ & - 6 vitaminas, hierro, calcio y cereales \\
\hline LLAOLLAO & HELADO LLAOLLAO & Natural Frozen Yogurt \\
\hline \multirow[t]{2}{*}{ HERO BABY } & HERO GUISANTES & $\begin{array}{ll}\text { - } & \text { Nuevos snacks de guisantes y maíz } 100 \% \text { ecológicos } \\
\text { - } & \text { Cereales y legumbres } 100 \% \text { ecológicos } \\
\text { - } & \text { NCO y BIO } \\
\text { - } & \text { Sin sal }\end{array}$ \\
\hline & HERO LENTEJAS & $\begin{array}{l}\text { - } \quad \text { Nuevos snacks de lentejas y maíz } 100 \% \text { ecológicos } \\
\text { - } \quad \text { Cereales y legumbres } 100 \% \text { ecológicos } \\
\text { ECO y BIO } \\
\text { - No fritos } \\
\text { - Sin sal }\end{array}$ \\
\hline \multirow[t]{2}{*}{ MCDONALD'S } & MCDONALD'S 1 & $\begin{array}{l}\text { - } \quad \text { Zumos sin azúcares añadidos } \\
\text { - } \quad \text { Zumo de manzana y zumo de naranja sin azúcares } \\
\text { añadidos }\end{array}$ \\
\hline & MCDONALD'S 2 & - $\quad$ Zumos sin azúcares añadidos \\
\hline
\end{tabular}




\begin{tabular}{|c|c|c|c|}
\hline & & & $\begin{array}{l}\text { Zumo de manzana y zumo de naranja sin azúcares } \\
\text { añadidos }\end{array}$ \\
\hline & MCDONALD'S 3 & $\bullet$ & Toma frutas y verduras cada día \\
\hline & MCDONALD'S 4 & • & Toma frutas y verduras cada día \\
\hline & MCDONALD'S 5 & $\bullet$ & Toma frutas y verduras cada día \\
\hline & MCDONALD'S 6 & $\bullet$ & Toma frutas y verduras cada día \\
\hline BABYBEL & QUESO BABYBEL 2 & $\bullet$ & $\begin{array}{l}\text { Sin conservantes } \\
\text { Sin colorantes añadidos }\end{array}$ \\
\hline STRINGS AND THINGS & QUESO CHEESTRINGS & • & $\begin{array}{l}\text { Cada cheestrings está hecho con un vaso de leche de } \\
180 \mathrm{ml}\end{array}$ \\
\hline \multirow[t]{2}{*}{ ALDI } & SUPERMERCADO ALDI 1 & $\bullet$ & $\begin{array}{l}\text { Pan recién hecho } \\
\text { Productos Eco }\end{array}$ \\
\hline & SUPERMERCADO ALDI 2 & & $\begin{array}{l}\text { Helados eco } \\
\text { Tarrinas GutBio }\end{array}$ \\
\hline VICHY CATALÁN & VICHY CATALÁN & & $\begin{array}{l}\text { Sin azúcares añadidos } \\
\text { Con estevia } \\
\text { Bebida a base de concentrado de zumo }\end{array}$ \\
\hline \multirow[t]{2}{*}{ DON SIMÓN } & ZUMO DON SIMÓN & & $\begin{array}{l}\text { Sabor natural } \\
\text { Solo zumo } 100 \% \text { exprimido }\end{array}$ \\
\hline & BEBIDA SIMON LIFE & & $\begin{array}{l}\text { Tiene vitaminas y no tiene gas } \\
\text { Es sano y natural } \\
\text { Sin conservantes } \\
\text { Vitaminas } B, C \text { y } E \\
\text { Sin gas } \\
\text { Contiene vitaminas } C \text { y } E \text { que contribuyen a la protección } \\
\text { de las células frente al daño oxidativo }\end{array}$ \\
\hline
\end{tabular}

Por último, resaltan las escasas referencias al contexto escolar en anuncios dirigidos al grupo 4-12 años (tan solo es explícito en tres anuncios). Este tipo de contenidos se muestra con enfoques muy distintos en los anuncios de zumo Don Simón, queso Cheestrings y galletas TostaRica Oceanix (tabla 7). En este caso, el anuncio del queso Cheestrings se articula por completo alrededor del contexto escolar, usando casi exclusivamente aspectos sociales y emocionales en su mensaje.

Tabla 7. Contenidos relacionados con el contexto escolar en los anuncios analizados.

\begin{tabular}{|c|c|c|}
\hline $\begin{array}{c}\text { MARCAS } \\
\text { ALIMENTARIAS }\end{array}$ & ANUNCIO & RELACIÓN CON EL CONTEXTO ESCOLAR \\
\hline DON SIMÓN & ZUMO DON SIMÓN & El niño que transmite el mensaje dice que el zumo es para llevar al cole. \\
\hline TOSTARICA & $\begin{array}{l}\text { GALLETAS TOSTARICA } \\
\text { OCEANIX }\end{array}$ & $\begin{array}{l}\text { Aparecen dibujos animados con mochilas en la espalda simulando que van al } \\
\text { cole. Ellos y la voz adulta masculina que transmite el mensaje dicen que con } \\
\text { esas galletas es más fácil aprender y cantar en inglés }\end{array}$ \\
\hline $\begin{array}{l}\text { STRINGS AND } \\
\text { THINGS }\end{array}$ & QUESO CHEESTRINGS & $\begin{array}{l}\text { El niño que transmite el mensaje dice que es el snack perfecto para tomar en la } \\
\text { hora del recreo. Aparecen imágenes del niño en clase mirando el reloj que hay } \\
\text { colgado en la pared esperando a que suene la campana para la hora del recreo. } \\
\text { Cuando suena la campana, aparece el niño cogiendo el queso cheestrings y } \\
\text { apuntando hacia el reloj. } \\
\text { Después se ven dos niñas en el patio del recreo y una de llevas lleva el queso } \\
\text { cheestrings y lo rompe en tiras. Aparecen también otros niños deshilachándolo y } \\
\text { comiéndoselo. } \\
\text { Una niña mira la imagen del envoltorio del queso y pinta el dibujo en el suelo del } \\
\text { patio del recreo junto con otro niño. } \\
\text { Niños y niñas jugando al fútbol y al acabar el partido deshilachan queso } \\
\text { cheestrings y se lo comen (todos los niños y niñas que aparecen muestran caras } \\
\text { de felicidad y diversión). }\end{array}$ \\
\hline
\end{tabular}

\section{Discusión}

El análisis de los espacios publicitarios de tres canales de televisión dirigidos al público infantil de edades comprendidas entre 4 y 12 años a lo largo de tres franjas horarias ha permitido identificar hasta 20 marcas distintas de productos alimentarios manifestadas en un total de 36 anuncios. De los tres canales infantiles analizados, Boing y Disney son los que más marcas alimentarias han emitido, alcanzado su máximo en los horarios de 17-19h y 20:30-22h del mes de julio. Esto supone la presencia de hasta 14 marcas de alimentación distintas en la misma franja horaria de dos horas (o menos) de algunos canales infantiles. Dentro de las 20 marcas alimentarias identificadas, McDonald's es la única que aparece en todos los canales y periodos estudiados, mientras el resto se anuncian principalmente durante los meses de marzo y julio de 2019, siendo en este último periodo donde se alcanza el máximo (un total de 14 marcas). La reducción de publicidad de marcas alimentarias durante el mes de noviembre (tan solo 3 marcas) 
coincide con el incremento, en el mismo espacio publicitario de estas cadenas, de la publicidad de juguetes correspondientes a la campaña prenavideña. Hay que señalar que precisamente en esos espacios del mes de noviembre aparecen dos marcas (Choco Krispies y Lanjarón) que no vuelven a aparecer en marzo y julio. Del mismo modo, algunos anuncios de helados y refrescos tan solo aparecen en los espacios publicitarios del verano (Frigo, LlaoLLao, Puleva, Vichy Catalán).

En cuanto al contenido de los anuncios analizados, lo primero que llama la atención es el uso de personajes famosos en la tercera parte de los mismos (12 de 36), en contra de lo que establece el Código PAOS de corregulación de la publicidad de alimentos y bebidas dirigida a menores, prevención de la obesidad y salud. Del mismo modo, el código trata de evitar que la publicidad resalte como particulares de un producto determinadas características que son comunes en ese tipo de producto. En este caso, resaltar determinados nutrientes y relacionarlos con necesidades vitales de las personas, como se observa en varios de los anuncios analizados, puede resultar confuso e incluso llegar a confundir a los niños (y a sus padres) sobre los posibles beneficios que aporta ese producto en concreto frente a otros de similares características, tal y como indicaban Cabello et al. (2016) o Bosqued et al. (2016) en trabajos anteriores. En cualquier caso, el ámbito de aplicación del Código PAOS no tiene un carácter normativo general y únicamente compromete a su cumplimiento a las empresas de los sectores político, alimentario y publicitario que firmaron el compromiso de adscripción.

Sin entrar a calificar los productos anunciados como saludables o no saludables, es evidente la gran cantidad de publicidad alimentaria que se emite en los canales de televisión que van dirigidos hacia el público infantil. Dada la influencia ejercida con su emisión en la televisión en los hábitos alimentarios y decisiones de compra de los niños, la identificación de los mismos y el conocimiento de sus contenidos pueden ser de utilidad para la prevención del sobrepeso y la obesidad infantil a través de una respuesta coordinada por parte de las autoridades sanitarias y escolares, así como por el compromiso responsable del sector publicitario y los medios de comunicación.

\section{Referencias}

AECOSAN, Agencia Española de Consumo, Seguridad Alimentaria y Nutrición (2012). Código de corregulación de la publicidad de alimentos y bebidas dirigida a menores, prevención de la obesidad y salud (Código PAOS). Madrid: Agencia Española de Seguridad Alimentaria y Nutrición. Ministerio de Sanidad, Servicios Sociales e Igualdad.

AECOSAN, Agencia Española de Consumo, Seguridad Alimentaria y Nutrición (2019). Evaluación y seguimiento de la estrategia NAOS: conjunto mínimo de indicadores. Madrid: Agencia Española de Seguridad Alimentaria y Nutrición. Ministerio de Sanidad, Consumo y Bienestar Social.

AESAN, Agencia Española de Seguridad Alimentaria y Nutrición (2005). Estrategia NAOS. Invertir la tendencia de la obesidad. Estrategia para la nutrición, actividad física y prevención de la obesidad. Madrid: Agencia Española de Seguridad Alimentaria.

Bacardí-Gascón, M y Jiménez-Cruz, A (2015). TV Food advertising geared to children in Latin-American countries and Hispanics in the USA: a review. Nutrición Hospitalaria, 31(5), 1928-1935.
Bawake, RA, Fernández-Barrés, S, Navarrete-Muñoz, EM, González-Palacios, S, Guxens, M, Irizar, A, Lertxundi, A, Sunyer, J, Vioque, J, Schröder, H, Vrijheid, M y Romaguera, D (2019). Impact of lifestyle behaviors in early childhood on obesity and cardiometabolic risk in children: Results from the Spanish INMA birth cohort study. Pediatric Obesity, 2019, e12590: 1-15

Boronat Mundina, J (2005). Análisis de contenido. Posibilidades de aplicación en la investigación educativa. Revista Interuniversitaria de Formación de Profesorado, 19(2), 157174.

Bosqued, MJ, López, L, Moya, A y Royo, MA (2016). La publicidad alimentaria dirigida a menores en España. Alimentación, nutrición y salud, 23(1), 19-25.

Busquet, J y Reinares, P (2009). La audiencia infantil de televisión en España. Ni tan escasa ni tan uniforme. Telos, 81, 129-141.

Cabello, A, España, E y Blanco, A (2016). La competencia en alimentación. Barcelona: Editorial Octaedro. 
Caroli, M, Argentieri, L, Cardone, M y Masi, A (2004). Role of television in childhood obesity prevention. International Journal of Obesity, 28, 104-108.

Casas Esteve, R y Gómez Santos, SF (2016). Estudio sobre la situación de la obesidad infantil en España. Madrid: Instituto DKV de la Vida Saludable.

Díaz Ramírez, G, Souto-Gallardo, MC, Bacardí Gascón, M y Jiménez-Cruz, A (2011). Efecto de la publicidad de alimentos anunciados en la televisión sobre la preferencia y el consumo de alimentos: revisión sistemática. Nutrición Hospitalaria, 26(6), 1250-1255.

Fernández-Gómez, E y Díaz-Campo, J (2014). La publicidad de alimentos en la televisión infantil en España: promoción de hábitos de vida saludables. Observatorio (OBS*) Journal, 8(4), 133-150.

González, C y López, JA (2012). La influencia de la publicidad infantil de alimentos: antecedentes y estado de la cuestión. Doxa Comunicación, 17, 119-142.

Gootman, J, McGinnis, J y Kraak, V (2006). Food Marketing to Children and Youth: Threat or Opportunity? Institute of Medicine. Washington, DC: National Academies Press.

Herrero Aguado, C (2008). El horario infantil en televisión: de la falta de imaginación a la irresponsabilidad de los mensajes publicitarios. Trastornos de la conducta alimentaria, 7, 751766.

Jiménez, M (2006). Cuando Barbie se come a Garfield. Publicidad y alimentación: niños obesos buscando la perfección del cuerpo adulto. Trastornos de la Conducta Alimentaria, 3, 245263.

Kelly, B, Jason CG, Halford, EJ, Boyland, KC, Bautista-Castaño, I, Berg, C, Caroli, M, Cook, B, Coutinho, JG, Effertz, T, Grammatikaki, E, Keller, K, Leung, R, Med, M, Manios, Y, Monteiro, R, Pedley, C, Prell, H, Raine, K, Recine, E, SerraMajem, L, Singh, S y Summerbell, C (2010). Television Food Advertising to Children: A Global Perspective. American Journal of Public Health, 100(9), 1730-1736.

León-Flández, KA (2017). Evaluación de la publicidad alimentaria dirigida a niños por televisión en España. Tesis doctoral. Universidad Complutense de Madrid.

León-Flández, K, Rico-Gómez, A, Moya-Geromín, MA, RomeroFernández, M, Bosqued-Estefanía, MJ, Damián, J, LópezJurado, L y Royo-Bordonada, MA (2017). Evaluation of compliance with the Spanish Code of self-regulation of food and drinks advertising directed at children under the age of 12 years in Spain, 2012. Public Health, 150, 121-129.

López Noguero, $F$ (2002). El análisis de contenido como método de investigación. XXI, Revista de Educación, 4, 167-179.

Martín, M, Fernández, MD y Ortiz, R (2011). Cinco años de Código PAOS en España: un análisis DAFO. Revista de Comunicación y Salud, 1(1), 33-43.

Moreno Castro, C (2006). Ingredientes mágicos y test clínicos en los anuncios como estrategias publicitarias. Comunicar: Revista Científica de Comunicación y Educación, 27(31), 123128.
OCU, Organización de Consumidores y Usuarios (2010). Publicidad de alimentos en TV. OCU-SALUD, 32-34.

Romero-Fernández, MM, Royo-Bordonada, MA y RodríguezArtalejo, F (2013). Evaluation of food and beverage television advertising during children's viewing time in Spain using the UK nutrient profile model. Public Health Nutrition, 16(7), 13141320.

Royo-Bordonada, MA, Bosqued-Estefanía, MJ, Damián, J, LópezJurado, L, Moya-Geromini, MA (2016). Nutrition and health claims in products directed at children via television in Spain in 2012. Gaceta Sanitaria, 30(3), 221-226.

Santos, M (2015). Ideas previas sobre alimentación en relación con las apetencias y el consumo de alimentos que llevan a cabo niños y niñas de 4 años. Trabajo de investigación educativa. Universidad de Granada.

Teruel Benitez, S (2014). Influencia de la publicidad televisiva en los menores. Análisis de las Campañas "Vuelta al cole" y "Navidad". Tesis Doctoral. Universidad de Málaga.

Tirado, F, Barbancho, FJ, Prieto, J y Moreno, A (2004). Influencia de los hábitos televisivos infantiles sobre la alimentación y el sobrepeso (II). Revista Cubana de Enfermería, 20(3).

Torres-García, M, Marrero-Montelongo, M, Navarro-Rodriguez, C y Gavidia, V (2017). ¿Cómo abordan los textos de Educación Primaria la competencia en alimentación y actividad física? Revista Eureka sobre Enseñanza y Divulgación de las Ciencias, 15(1), 1103.1-16.

Vik, FN, Bjørnarå, HB, Overby, NC, Lien, N, Androutsos, O, Maes, L, Jan, N, Kovacs, E, Moreno, LA, Dössegger, A, Manios, Y, Brug, J y Bere, $E$ (2013). Associations between eating meals, watching TV while eating meals and weight status among children, ages 10-12 years in eight European countries: the ENERGY cross-sectional study. International Journal of Behavioral Nutrition and Physical Activity, 10(58), 1-10. 